\title{
Impact of the long chain $\omega$-acylceramides on the stratum corneum lipid nanostructure. Part 1: Thermotropic phase behaviour of CER[EOS] and CER[EOP] studied using X-ray powder diffraction and FT-Raman spectroscopy
}

\author{
Doreen Kessner ${ }^{\mathrm{a}}$, Gerald Brezesinski ${ }^{\mathrm{b}}$, Sergio S. Funari ${ }^{\mathrm{c}}$, Bodo Dobner ${ }^{\mathrm{a}}$, Reinhard H.H. Neubert ${ }^{\mathrm{a}, *}$ \\ a Institute of Pharmacy, Martin-Luther-University, Wolfgang-Langenbeck-Straße 4, 06120 Halle, Germany \\ ${ }^{\mathrm{b}}$ Max Planck Institute of Colloids and Interfaces, Research Campus Golm, 14476 Potsdam, Germany \\ ${ }^{\text {c } H A S Y L A B}$ at DESY, Notkestr. 85, 22603 Hamburg, Germany
}

\section{A R T I C L E I N F O}

\section{Article history:}

Received 17 March 2009

Received in revised form 12 October 2009

Accepted 26 October 2009

Available online 10 November 2009

\section{Keywords:}

Stratum corneum

Ceramide [EOS]

Ceramide [EOP]

X-ray powder diffraction

FT-Raman spectroscopy

\begin{abstract}
A B S T R A C T
The stratum corneum (SC), the outermost layer of the mammalian skin, is the main skin barrier. Ceramides (CERs) as the major constituent of the SC lipid matrix are of particular interest. At the moment, 11 classes of CERs are identified, but the effect of each single ceramide species is still not known.

Therefore in this article, the thermotropic behaviour of the long chain $\omega$-acylceramides CER[EOS] and CER[EOP] was studied using X-ray powder diffraction and FT-Raman spectroscopy.

It was found that the $\omega$-acylceramides CER[EOS] and CER[EOP] do not show a pronounced polymorphism which is observed for shorter chain ceramides as a significant feature. The phase behaviour of both ceramides is strongly influenced by the extremely long acyl-chain residue. The latter has a much stronger influence compared with the structure of the polar head group, which is discussed as extremely important for the appearance of a rich polymorphism. Despite the strong influence of the long chain, the additional OH-group of the phyto-sphingosine type CER[EOP] influences the lamellar repeat distance and the chain packing. The less polar sphingosine type CER[EOS] is stronger influenced by the long acyl-chain residue. Hydration is necessary for the formation of an extended hydrogen-bonding network between the polar head groups leading to the appearance of a long-periodicity phase (LPP). In contrast, the more polar CER[EOP] forms the LPP with densely packed alkyl chains already in the dry state.
\end{abstract}

(c) 2009 Elsevier Ireland Ltd. All rights reserved.

\section{Introduction}

The outermost layer of the skin, the stratum corneum (SC), is the major barrier both to dermal and to transdermal delivered drugs and plays a key role in the skin barrier integrity (Wertz and van den Bergh, 1998). This superficial layer consists of dead cells, the corneocytes, filled with keratin, which are embedded in a complex matrix of multilamellar organized lipids (Elias, 1983). The unique lipid composition is one important feature of the SC. The major lipid classes are ceramides (CER), cholesterol, and long chain free fatty acids (Gray and Yardley, 1975; Gray and White, 1978). It is generally accepted that ceramides as the main constituents play a predominant role in maintaining the barrier function (Holleran et al., 1991; Coderch et al., 2003). Therefore, it becomes apparent that increasing interest is focussed on this particular class of lipids.

* Corresponding author at: Institute of Pharmacy, Martin-Luther-University Halle-Wittenberg, Wolfgang-Langenbeck-Str. 4, DE-06120 Halle(Saale), Germany. Tel.: +49345 5525000; fax: +493455527292.

E-mail address: reinhard.neubert@pharmazie.uni-halle.de (R.H.H. Neubert).
To date, 11 ceramide subclasses have been detected in the SC lipid matrix (Mizutani et al., 2009).

Ceramides consist of a long chain fatty acid bound to the aminogroup of a long chain di- or trihydroxy sphingoid base (sphingosine, phyto-sphingosine, and 6-hydroxysphinganine). The acyl residue of ceramides can be hydroxylated at the $\alpha$-position or at the end of the hydrocarbon chain ( $\omega$-position) (Fig. 1$)$.

Up to now, a detailed picture of the molecular organization of lipids in the SC, in particular the function of each ceramide subclass, has not been fully elucidated. It is clear that a profound knowledge of the physical properties of the SC lipids and of their interactions is essential for a deeper understanding of the impact of each ceramide species on the barrier function of the SC.

The physical properties of SC lipids were studied using a variety of experimental techniques such as small angle X-ray diffraction (Friberg and Osborne, 1985; Bouwstra et al., 2000), differential scanning calorimetry (Bouwstra et al., 1989; Shah et al., 1995; Wegener et al., 1996), electron microscopy (Swartzendruber et al., 1989) and vibrational spectroscopy as Fourier transform infrared (FT-IR) (Moore et al., 1997; Lafleur, 1998; Mendelsohn and Moore, 1998; Chen et al., 2000) and FT-Raman spectroscopy (Neubert et 


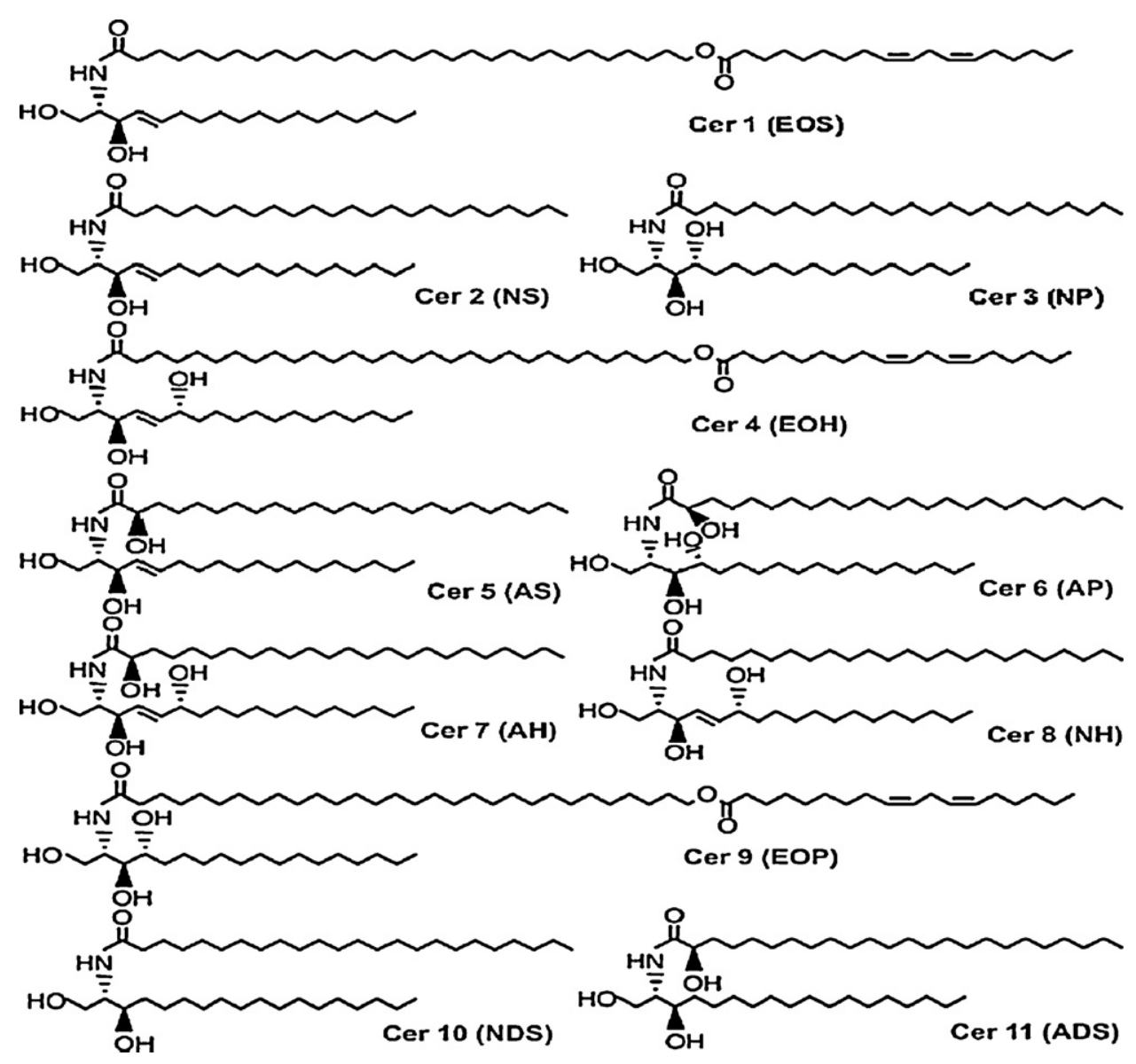

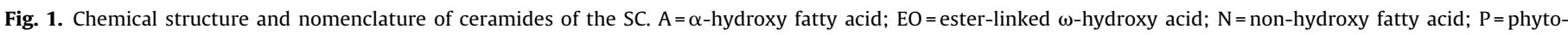
sphingosine; $\mathrm{S}=$ sphingosine; $\mathrm{H}=6$-hydroxysphingosine; $\mathrm{D}=$ dihydro according to Mizutani et al. (2009).

al., 1997; Wegener et al., 1997; Wartewig et al., 1998; Raudenkolb et al., 2003b).

Summarizing, a rich polymorphism appears to be a characteristic feature of ceramides and seems to be important for the function of the SC lipid matrix. At physiological temperatures, all crystalline phases of ceramides exhibit lamellar structures with highly ordered hydrocarbon chains. Among the different phases of the ceramides, those with fully extended molecules packed in a double layer with either their two chains parallel oriented or stretched out to opposite direction of the head group are thought to be of particular relevance for the molecular architecture of the SC lipid matrix. The different behaviour of the ceramide head groups may be an important factor for the skin barrier function.

Unfortunately, data about the physicochemical properties of the acylceramides CER[EOS] and CER[EOP], which seem to be crucial for the integrity of the SC lipid matrix, are lacking so far because the availability of these compounds is limited.

From X-ray diffraction studies on some SC lipid model systems containing CER[EOS] it was concluded that CER[EOS] is a prerequisite for the formation of the long-periodicity phase (LPP) with $13 \mathrm{~nm}$ (Bouwstra et al., 2000; McIntosh et al., 1996; McIntosh, 2003; Jager et al., 2005). In order to determine the influence of the head group structure, CER[EOS] was partially replaced by CER[EOP] which contains an additional hydroxyl group at the sphingoid backbone. It can be stated that the head group structure has a remarkable influence on the behaviour and structure of the SC lipids. The substitution of CER[EOS] by CER[EOP] results in a reduction of the LPP and in a phase separation which was attributed to the larger and more hydrophilic head group of CER[EOP] (Jager et al., 2004).
However, the existence of the $13 \mathrm{~nm}$ lamellar repeat pattern in stratum corneum in vivo is currently under discussion. Apart from some conventional electron micrographs (Swartzendruber et al., 1989), the $13 \mathrm{~nm}$ repeat unit has been observed in some SAXD studies (White et al., 1988; Bouwstra et al., 1991; Hatta et al., 2001), while it has not been confirmed in other SAXD studies (Garson et al., 1991) or in cryo-transmission electron microscopy studies on native hydrated epidermis samples (Al-Amoudi et al., 2005) or in neutron diffraction studies on hydrated stratum corneum (Charalambopoulou, 2004).

In the work of Kessner et al. (2008), the analysis of neutron diffraction patterns of a CER[EOS]/CER[AP]/CHOL model membrane implies that one CER[EOS] molecule penetrates from one membrane layer into an adjacent layer. A $13 \mathrm{~nm}$ periodicity phase has not been observed under the experimental conditions used in these experiments. CER[EOS] can be arranged inside a phase with a repeat distance of $4.5 \mathrm{~nm}$ which is predominantly formed by short-chain CER[AP] with distinct polarity.

In the present work, we report a comprehensive analysis of the thermotropic behaviour of CER[EOS] and CER[EOP] by means of $\mathrm{X}$-ray powder diffraction and FT-Raman spectroscopy.

\section{Materials and methods}

\subsection{Materials}

CER[EOS] and CER[EOP] were generously provided by Evonik Goldschmidt GmbH (Essen, Germany). In order to increase chemical purity above $96 \%$, the substances were treated using a MPLC technique on a silica gel column with a chloroform/methanol 
gradient. The substances were crystallised from the solvent, respectively. The identity of both ceramides $($ CER $[E O S]=1012 \mathrm{~g} / \mathrm{mol}$; CER $[E O P]=1028 \mathrm{~g} / \mathrm{mol}$ ) was proved by mass spectrometry.

\subsection{X-ray diffraction}

The X-ray powder diffraction measurements were carried out at the soft condensed matter beamline $A 2$ at storage ring Doris III of HASYLAB (DESY, Hamburg, Germany). The $\omega$-acylceramides CER[EOS] and CER[EOP] were studied in dry and hydrated state, respectively. For obtaining X-ray powder diffraction patterns of the ceramides in dry state, about $2 \mathrm{mg}$ of CER[EOS] and CER[EOP] as received from purification, respectively, were placed in sealed $\mathrm{X}$ ray glass capillaries (Hilgenberg $\mathrm{GmbH}$, Malsfeld, Germany) with a diameter of $1 \mathrm{~mm}$ and a wall thickness of $0.1 \mathrm{~mm}$.

For studying the influence of hydration, the ceramide was dispersed in water in order to obtain a ceramide/water-mixture ( $20 \mathrm{wt} \%$ of ceramide in water). The hydrated samples were also placed in sealed glass capillaries and equilibrated for $48 \mathrm{~h}$ at room temperature.

The samples of each ceramide at dry and hydrated state were fixed in a temperature-controlled sample holder that was heated/cooled, respectively. A monochromatic X-ray beam with a wavelength of $0.150 \mathrm{~nm}$ was used. The diffraction intensities were detected by two linear position-sensitive detectors (PSD) in the small- and wide-angle regions (SAXS: $0<s<0.5 \mathrm{~nm}^{-1}$, WAXS: $1.8<s<3 \mathrm{~nm}^{-1}$ ) simultaneously. The temperature dependence of the X-ray diffraction patterns was studied in heating/cooling cycles: CER[EOS]-dry state: $20-90^{\circ} \mathrm{C}$; hydrated state: $20-80^{\circ} \mathrm{C}$; CER[EOP]-dry state: $20-100^{\circ} \mathrm{C}$; hydrated state: $20-96^{\circ} \mathrm{C}$. After each temperature step, the sample has been allowed to equilibrate for $5 \mathrm{~min}$ before the diffraction pattern was recorded. To minimize the X-ray exposure on the sample, a shutter mounted before the sample was kept closed when no data were acquired. From the peak maximum positions, the repeat distances $d$ were obtained after calibration with standards: dry rat-tail collagen and $p$-bromobenzoic acid for the SAXS and the WAXS regions, respectively. Each diffraction pattern is presented here as scattering intensity $I_{\text {corr }}(\theta)$ in arbitrary units versus the reciprocal spacing $s(s=(2 \sin \theta) / \lambda)$.

\subsection{Fourier transform Raman spectroscopy}

FT-Raman spectra were recorded with a Bruker Fourier transform infrared spectrometer RFS 100/S (Bruker Optics, Ettlingen, Germany). A diode pumped Nd:YAG laser which emits radiation at $1064 \mathrm{~nm}$ was used as the excitation source. The scattered radiation was collected at $180^{\circ}$ to the source. Typical spectra were recorded at a laser power of $300 \mathrm{~mW}$ at sample location and a resolution of $4 \mathrm{~cm}^{-1}$.
The samples were placed in X-ray capillaries and sealed airproof. The temperature dependence of the Raman spectra of CER[EOS] was studied in the range from 20 to $90^{\circ} \mathrm{C}$ and of CER[EOP] in the range from 25 to $115^{\circ} \mathrm{C}$. Thereby, the temperature was monitored using a thermocouple located at the capillary near to the sample. The samples were allowed to equilibrate for $5 \mathrm{~min}$ to equilibrate before recording a spectrum. The evaluation of the spectra was carried out using the Bruker OPUS software package. Generally, Raman intensities were determined as integrated band intensities.

\section{Results and discussion}

\subsection{X-ray diffraction studies of CER[EOS]}

First, X-ray diffraction patterns of dry CER[EOS] were collected. At $20^{\circ} \mathrm{C}$, the sample exhibits only two equidistant Bragg peaks in the SAXS region indicating the appearance of a lamellar gel phase (Fig. 2). With increasing temperature, the reflections become sharper and more pronounced showing a better correlation between the lamellar sheets, before they finally disappear at the melting point $\left(86^{\circ} \mathrm{C}\right)$.

In the WAXS region, one Bragg peak was identified below the melting point which was indicative of a hexagonal packing of ordered hydrocarbon chains (Fig. 2). The corresponding crosssectional area of the chains, calculated from that reflection equals $0.204 \mathrm{~nm}^{2}$ at $20^{\circ} \mathrm{C}$ and increased to $0.21 \mathrm{~nm}^{2}$ close to the melting temperature. This Bragg peak transforms into a broad halo at the melting point characteristic for molten hydrocarbon chains. On cooling the sample, the lamellar gel phase is re-formed at $76^{\circ} \mathrm{C}$, with even higher intensity and more pronounced higher order Bragg peaks. The WAXS region exhibits now two sharp reflections indicative of an orthorhombic packing of the hydrocarbon chains. The chain packing depends obviously on the sample history. Crystallization from the solvent or from the melt leads to different structures. A second heating scan confirms these findings leading to the following results:

(i) The thermotropic phase behaviour of CER[EOS] in dry state is reversible with a certain hysteresis between heating and cooling. Melting the sample does not lead to thermal decomposition of the sensitive un-saturated linoleic acid residue.

(ii) In the molten state, no superstructure was observed. This shows that the sample does not form a liquid-crystalline state but rather a real melt maybe characterized by larger aggregates or micelles.

(iii) The thermal history influences the chain packing. After recrystallization during cooling, an orthorhombic lattice is observed instead of the hexagonal packing observed on first heating.

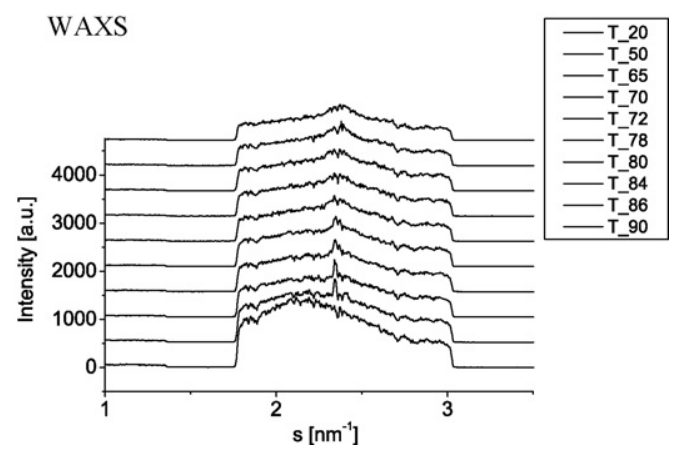

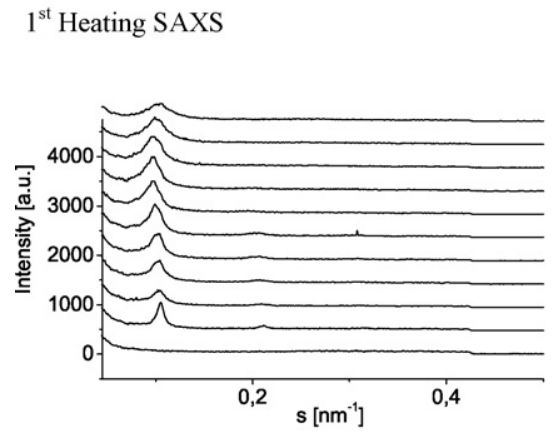

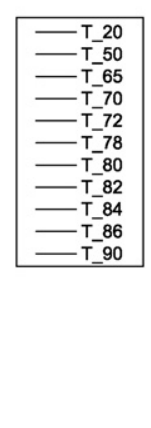

Fig. 2. X-ray diffraction patterns of dry CER[EOS] taken at different temperatures (indicated). 


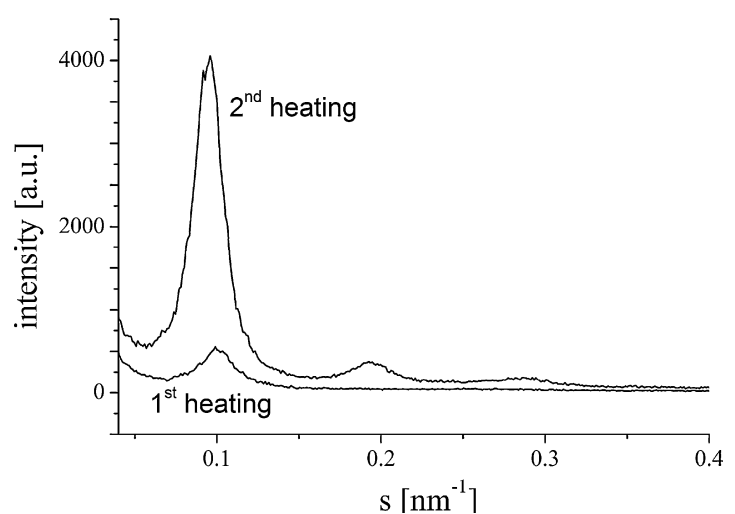

Fig. 3. X-ray diffraction patterns of dry CER[EOS] taken at $50^{\circ} \mathrm{C}$ in the first and second heating scans.

(iv) The lamellar repeat distance of $\sim 10 \mathrm{~nm}$ (the d-value is slightly larger in the second heating scan, e.g., $10.4 \mathrm{~nm}$ at $50^{\circ} \mathrm{C} \mathrm{com-}$ pared with $9.9 \mathrm{~nm}$ during the first heating, see Fig. 3) differs clearly from the often discussed $13 \mathrm{~nm}$ (Bouwstra et al., 2002). The shorter periodicity may be the result of a partial chain interdigitation of two opposite CER[EOS] molecules.

Additionally, CER[EOS] was studied in a mixture with water to investigate the influence of hydration on the structural arrangement (Fig. 4). At $20^{\circ} \mathrm{C}$, the sample exhibits several Bragg peaks in the SAXS region. The $s$-values are in a ratio of $1: 2: 3: 4$ indicating the appearance of a highly ordered lamellar phase. It is interesting to note that the 3rd order peak is hardly visible whereas the 4th order peak has a pronounced intensity. The $d$-value amounts to approximately $12.6 \mathrm{~nm}$ indicating the formation of a LPP. With increasing temperature, the Bragg peaks shift only slightly to smaller $s$-values. The melting of this highly crystalline phase starts at $\sim 74^{\circ} \mathrm{C}$, where the coexistence of two phases can be observed. The phase with the smaller $d$-value $(9.6 \mathrm{~nm})$ is still observable at $76^{\circ} \mathrm{C}$ but disappeared completely at $78^{\circ} \mathrm{C}$. In the WAXS patterns several Bragg peaks were observed indicative of a crystalline packing of ordered hydrocarbon chains. Above the melting point, these Bragg peaks transform into a broad halo characteristic for molten hydrocarbon chains.

On cooling the sample, the lamellar gel phase was re-formed, whereby the Bragg peaks corresponding to the two phases (one with larger and one with smaller $d$-values) are seen at all temperatures.

Summarising the results, the compound does not form any periodic structure above the melting point. The larger $d$-spacing in the gel phase compared to the dry state arises from the hydration of the sample resulting in a water layer between the lipid layers or in a different arrangement of the molecules. On cooling and re-heating the hydrated sample, phases with different $d$-values coexist. The smaller $d$-value of one of these phases is similar to the one observed for the dry sample. The decrease in the melting temperature is the result of lipid hydration. Similar behaviour is also observed for many phospholipids.

\subsection{X-ray diffraction studies of CER[EOP]}

By comparison of the physical-chemical properties of CER[EOS] and CER[EOP] with an additional OH-group, the role of the head group polarity should be elucidated. X-ray diffraction studies on synthetic lipid mixtures containing CER[EOS] and/or CER[EOP] revealed differences in the formation of the lamellar lipid organisation in dependence on the composition ratio (Jager et al., 2004).

The SAXS patterns of CER[EOP] in dry state show clearly more Bragg peaks than the corresponding CER[EOS], even at room temperature (Fig. 5 ).

The lamellar repeat distance $d$ equals $12.5 \mathrm{~nm}$ (LPP), which is in the range of the molecular size of two opposing CER[EOP] molecules. Maybe because of the phyto-sphingosine head group with three neighbouring $\mathrm{OH}$-groups, lateral hydrogen bonds are
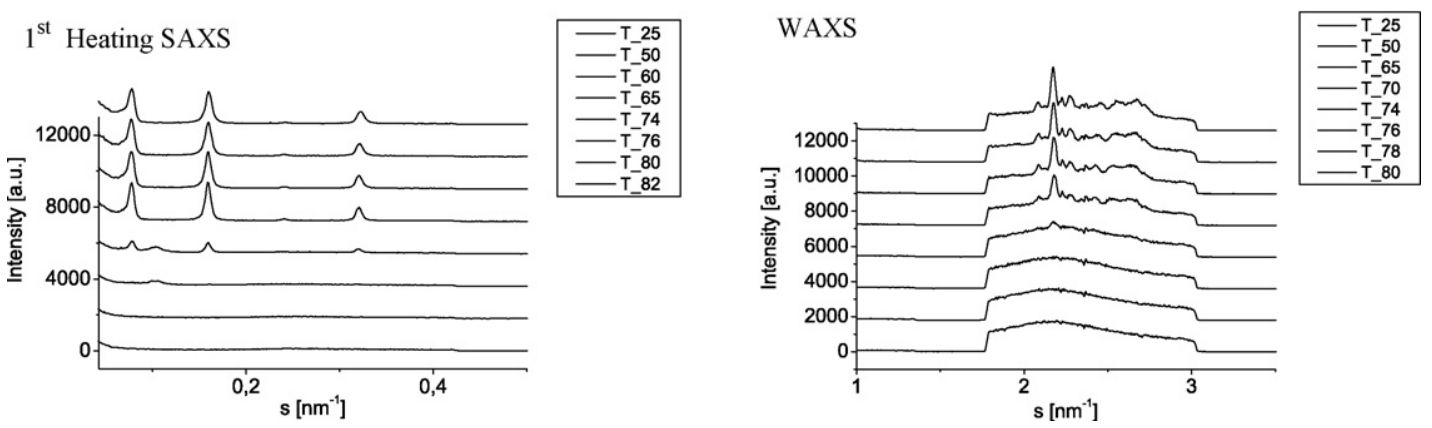

Fig. 4. X-ray diffraction patterns of hydrated CER[EOS] taken at different temperatures (indicated).
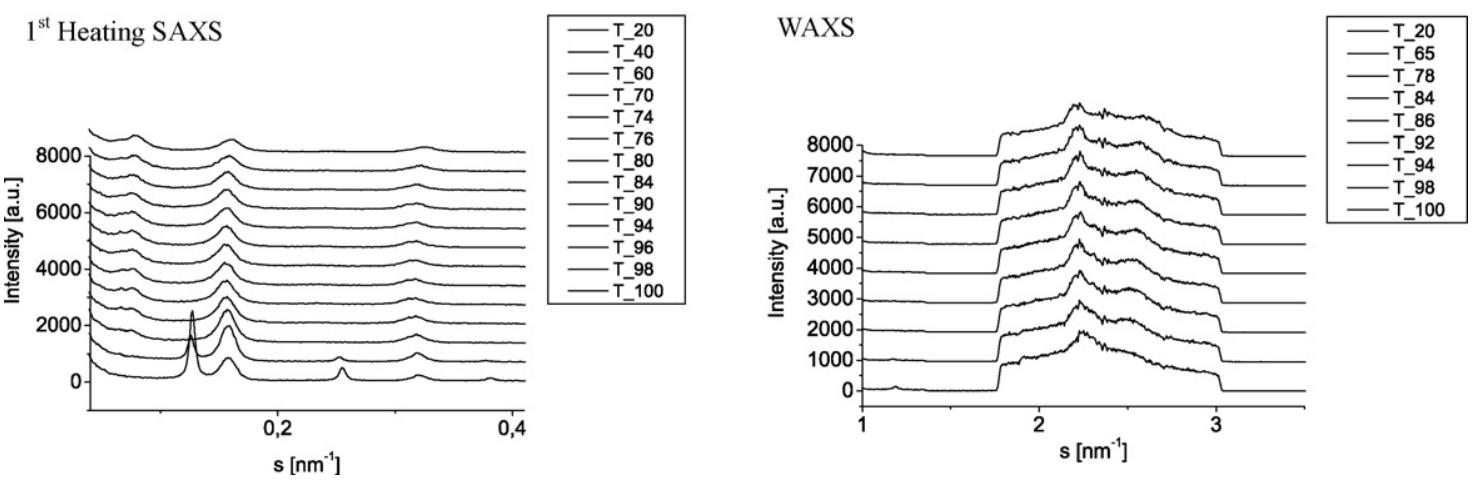

Fig. 5. X-ray diffraction patterns of dry CER[EOP] taken at different temperatures (indicated). 

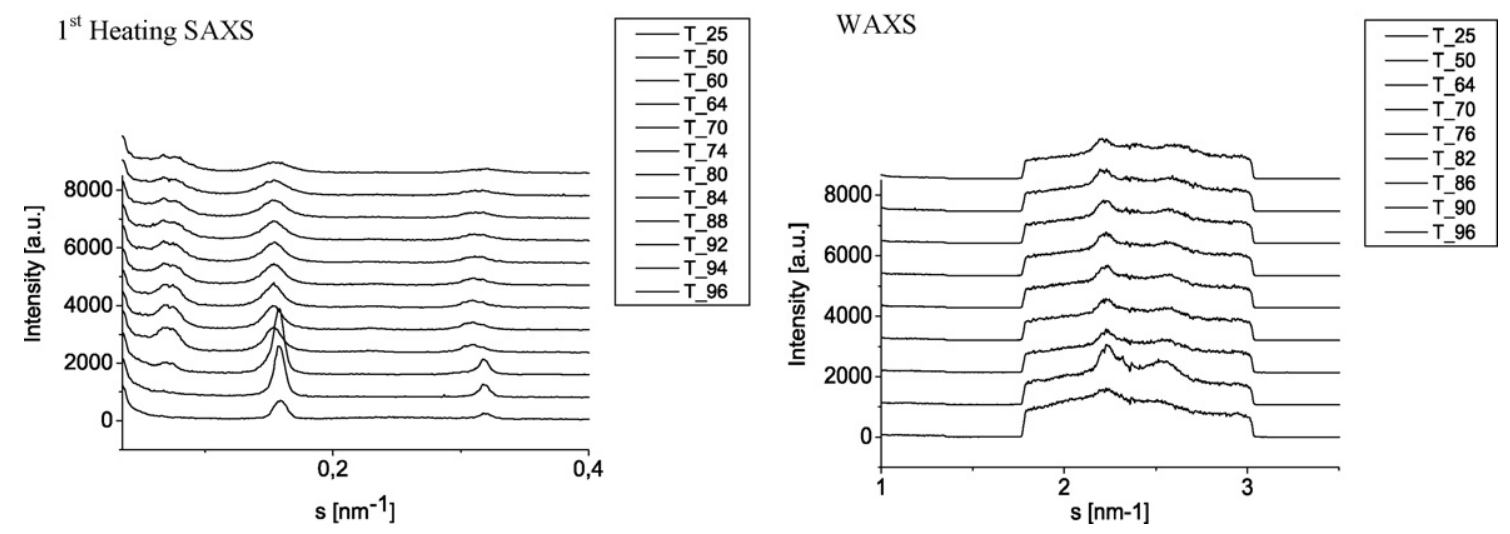

Fig. 6. X-ray diffraction patterns of hydrated CER[EOP] taken at different temperatures (indicated).

induced acting as a backbone of the lamellar systems. Therefore, the partial interdigitation of alkyl chains as discussed for dry CER[EOS], resulting in a shorter periodicity, has not been observed for CER[EOP].

From the SAXS patterns, it can be seen that the Bragg peaks became sharper and more pronounced with increasing temperature. Unfortunately, the melting point of CER[EOP] could not be detected due to the limited temperature range accessible at the beam line A2. This marks another important difference to dry CER[EOS] which revealed a melting point at $86^{\circ} \mathrm{C}$. It is known from DSC and Raman spectroscopy that the melting of CER[EOP] starts only at temperatures above $100^{\circ} \mathrm{C}$. This difference in melting temperatures should be caused by the different head group polarity as well. Because of additional $\mathrm{OH}$-groups, more hydrogen bonds are formed which require higher energy for the melting process.

Interestingly, the SAXS patterns reveal the appearance of a second phase with three equidistant peaks at $100^{\circ} \mathrm{C}$. The corresponding $d$-value equals $\sim 10 \mathrm{~nm}$. This phase correlates to the phase observed for CER[EOS] in dry state. Maybe, most of the hydrogen bonds are disbanded at $100^{\circ} \mathrm{C}$ and the new phase is composed of two opposing CER[EOP] molecules with a partial overlapping of the alkyl chains.

In the WAXS region, two reflections can be seen indicative of an orthorhombic chain packing. The Bragg peak with the larger $s$ value moves towards the first one with increasing temperature, indicating that the distortion of the lattice is reduced continuously.

On cooling the sample, the high-temperature phase disappeared finally at $91^{\circ} \mathrm{C}$. This behaviour shows that an extended hydrogen bond-network is re-formed on cooling.

Additionally, CER[EOP] was studied in mixtures with water in order to investigate the influence of hydration on the structural arrangement (Fig. 6).

The SAXS pattern at $25^{\circ} \mathrm{C}$ shows, that the sample exhibits several Bragg peaks in the ratio of 1:2:3:4 indicating the existence of a highly ordered lamellar gel phase with a $d$-spacing of $12.5 \mathrm{~nm}$. This phase is similar to the one observed in dry state showing that CER[EOP] requires no water for the formation of the LPP. This is an essential difference to CER[EOS].

With increasing temperature, the intensity of the Bragg peaks increased. Similar to the dry state, the melting point could not be reached. Interestingly, the high-temperature phase observed in dry state was not found in hydrated samples. Obviously, the head groups are always sufficiently hydrated avoiding the interdigitation of alkyl chains.

The WAXS patterns show two reflections indicative of an orthorhombic chain packing. Cooling the sample does not change the WAXS patterns. The second heating cycle confirms the observations summarized as follows: (i) The acylceramide head group architecture influences considerably the thermotropic phase behaviour. In contrast to CER[EOS], CER[EOP] forms even in dry state the LPP.

(ii) Because of a distinct intermolecular hydrogen bond-network, CER[EOP] possesses a melting point above $100^{\circ} \mathrm{C}$.

(iii) At $98^{\circ} \mathrm{C}$, a second high-temperature phase appears for CER[EOS] in dry state.

(iv) In dry as well as in hydrated state, the hydrocarbon chains of CER[EOP] show an orthorhombic packing.

In order to understand better the structuring of CER[EOP], the lateral chain packing was studied by analysing the WAXS patterns. For SC lipid organization three classes of lateral organization are important (Bouwstra and Ponec, 2006). A single reflexion at $0.41 \mathrm{~nm}$ indicates a hexagonal packing with a cross-section area of $0.194 \mathrm{~nm}^{2}$. Two reflexions at $0.41 \mathrm{~nm}$ and at $0.37 \mathrm{~nm}$ are indicative of an orthorhombic chain packing with an extremely small cross-section area of $0.182 \mathrm{~nm}^{2}$.

Concerning CER[EOP] in dry state, it was stated above, that the WAXS patterns display an orthorhombic chain packing indicated by Bragg peaks located at 0.45 and $0.38 \mathrm{~nm}$. In the temperature range between 20 and $98^{\circ} \mathrm{C}$, the $0.38 \mathrm{~nm}$ reflexion is shifted continuously to larger $d$-values, whereby the one at $0.45 \mathrm{~nm}$ is only slightly influenced. The shift of the two Bragg peaks indicates the tendency to reduce the distortion of the chain lattice at higher temperatures. Thereby, the lipids can rotate along their longest axis resulting in a lesser dense packing. The area per alkyl chain was calculated from the corresponding $s$-values taken from the WAXS patterns. At $20^{\circ} \mathrm{C}$, the area per alkyl chain of $0.187 \mathrm{~nm}^{2}$ shows the high packing density of an orthorhombic phase. With increasing temperature, the packing density decreases slightly. The chain cross-section increases to $0.19 \mathrm{~nm}^{2}$ at $98^{\circ} \mathrm{C}$.

The distortion of the lattice can be calculated from the semiaxis of the ellipse connecting six adjacent molecules (Bringezu et al., 2002). At $20^{\circ} \mathrm{C}$ and $98^{\circ} \mathrm{C}$, the distortion equals 0.2286262 and 0.183044 , respectively. The reduction of distortion with increasing temperature indicates the steady transition from an orthorhombic to a hexagonal lattice, because the latter is per se undistorted.

Summarising, the WAXS patterns of anhydrous CER[EOP] reflect an orthorhombic chain packing at $T<100^{\circ} \mathrm{C}$. The lattice distortion decreases with increasing temperature. The melting of the chains could not be observed due to limited accessible temperature range. The addition of water has a clear influence on the chain lattice. The head groups appear to hydrate easily, but no defined water layer between the ceramide layers was formed. Although the orthorhombic lattice still exists, the single chains require more space. The calculated cross-section area equals $0.192 \mathrm{~nm}^{2}$ after addition of water at $25^{\circ} \mathrm{C}$ and increases by $0.006 \mathrm{~nm}^{2}$ to $0.198 \mathrm{~nm}^{2}$ at $90^{\circ} \mathrm{C}$. 

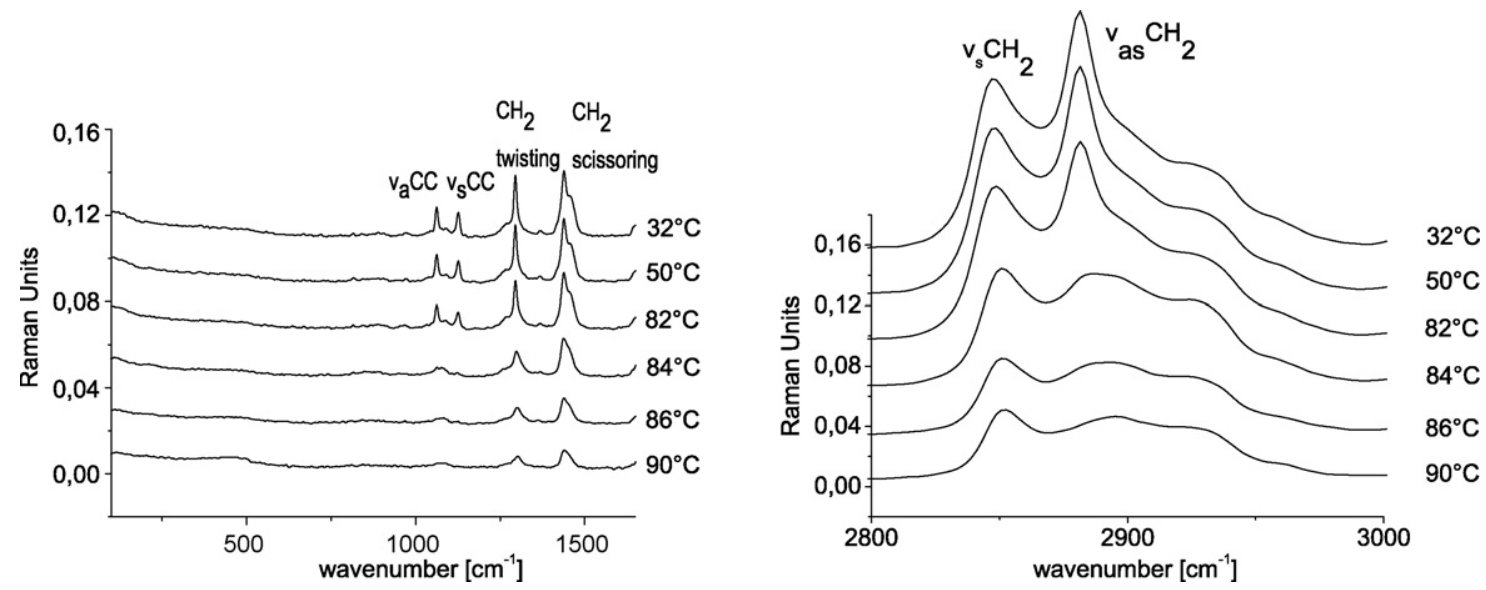

Fig. 7. Raman spectra of dry CER[EOS] in the spectral ranges: (left) $100-1650 \mathrm{~cm}^{-1}$ and (right) $2800-3000 \mathrm{~cm}^{-1}$ taken at different temperatures (indicated).

Finally, the X-ray diffraction studies on the $\omega$-acylceramides revealed that CER[EOS] as well as CER[EOP] form lamellar phases with a repeat distance of approx. $12.5 \mathrm{~nm}$, the so-called longperiodicity phase (LPP). Interestingly, CER[EOS] exhibits this characteristic phase only after hydration, whereas CER[EOP] shows the LPP even in dry state. The ability to form the LPP is a feature of both $\omega$-acylceramides.

All skin lipids exhibit an exceptional organization of lamellar phases, among the one with $12-13 \mathrm{~nm}$ periodicity (long periodicity phase-LPP) is considered to be crucial for the skin barrier properties (Bouwstra et al., 2000). In skin lipid mixtures used to reproduce in vitro the multilamellar lipid organization of SC (Jager et al., 2005), the presence of CER[EOS] has been regarded to be a prerequisite for the formation of the LPP. As stated above, the molecular organisation of the SC lipid matrix including the existence of the LPP is still a matter of strong debate. From $\omega$-acylceramides used to create a multilamellar structure it cannot be concluded that a skin lipid mixture induces the formation of a LPP in a similar. According to literature, the organization of the multilamellar SC lipid (model) membranes is influenced by a lot of factors (Swartzendruber et al., 1989; White et al., 1988; Bouwstra et al., 1991; Garson et al., 1991; Hatta et al., 2001; Al-Amoudi et al., 2005; Charalambopoulou, 2004; Kessner et al., 2008).

\subsection{Raman spectroscopy}

Utilization of Raman spectroscopy for elucidating phase transitions, the population of trans and gauche conformers, and the state of order as well as the packing properties of hydrocarbon chains of lipids is well established (Kirchoff and Levin, 1987; Mendelsohn and Moore, 1998; Raudenkolb et al., 2005b).

The Raman spectrum of anhydrous CER[EOS] at selected temperatures is shown in Fig. 7.

The assignments of particular bands according to literature data (Snyder and Scherer, 1979; Snyder et al., 1982; Mendelsohn and Moore, 1998; Wartewig and Neubert, 2007) are summarized in Table 1 . The major part of the Raman spectrum consists of bands related to the alkyl chain. The longitudinal acoustic mode (LAM) $\left(<300 \mathrm{~cm}^{-1}\right)$ indicates that the chains are in all-trans conformation, and the $\mathrm{CH}_{3}$ rocking mode $\left(890 \mathrm{~cm}^{-1}\right)$ shows chains with three or more trans bonds in a row. Interestingly, no LAM as well as no $\mathrm{CH}_{3}$ rocking mode was detected. Obviously, the chain end of CER[EOS] shows no all-trans conformation.

This effect is probably based on the unsaturated linoleic acid residue, which causes a high fluidity of the chain preventing a high packing density. Similar studies on pure linoleic acid prove these assumptions (data not shown). The splitting of the $\mathrm{CH}_{2}$ scissoring
Table 1

Selected Raman bands of anhydrous CER[EOS].

\begin{tabular}{ll}
\hline Band position $\left(\mathrm{cm}^{-1}\right)$ & Assignment \\
\hline 1063 & $v_{\text {as }}(\mathrm{C}-\mathrm{C})$, three or more trans bonds in sequence \\
1130 & $v_{\mathrm{s}}(\mathrm{C}-\mathrm{C})$, three or more trans bonds in sequence \\
1295 & $\mathrm{CH}_{2}$ twisting \\
$1439 ; 1458$ & $\mathrm{CH}_{2}$ scissoring \\
2848 & $v_{\mathrm{s}}\left(\mathrm{CH}_{2}\right)$ \\
2880 & $v_{\text {as }}\left(\mathrm{CH}_{2}\right)$, three or more trans bonds in sequence \\
\hline
\end{tabular}

mode into a doublet indicates an orthorhombic packing of the alkyl chains.

The appearance of $v_{\text {as }}\left(\mathrm{CH}_{2}\right), v_{\mathrm{s}}(\mathrm{C}-\mathrm{C}$ trans $)$ bands indicates the ordered structure of the hydrocarbon chains. The band position of the $\mathrm{CH}_{2}$ symmetric stretching mode $\left(v_{\mathrm{s}}\left(\mathrm{CH}_{2}\right)\right)$ is an indicator for the order of the hydrocarbon chains. The lower the band position is, the higher is the order (Mendelsohn and Moore, 1998; Raudenkolb et al., 2003b). The temperature dependence of the $v_{\mathrm{s}}\left(\mathrm{CH}_{2}\right)$ mode shows changes in the chain packing (Fig. 8 ) induced by the temperature.

At room temperature, the $v_{\mathrm{S}}\left(\mathrm{CH}_{2}\right)$ band is at $2846 \mathrm{~cm}^{-1}$, indicative of a densely packed structure (McPhail et al., 1984). Heating the sample shifts the band position continuously to higher wavenumbers. Between 80 and $90^{\circ} \mathrm{C}$, a drastic shift by more than $2.2 \mathrm{~cm}^{-1}$ is observed indicating a substantial loss of order during the phase transition. The final band position of $2851.9 \mathrm{~cm}^{-1}$ represents the molten chains with no defined order (Snyder et al., 1982). These results correspond well to the ones from X-ray diffraction studies. The slight differences in the melting point (X-ray: $86^{\circ} \mathrm{C}$ versus

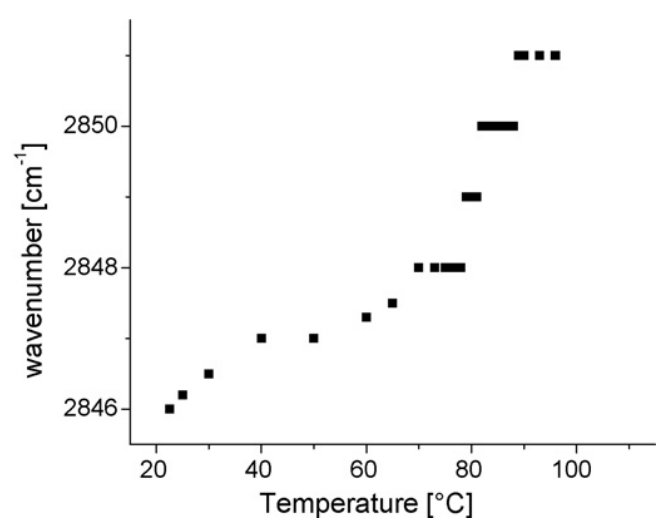

Fig. 8. Temperature dependence of the band position of the $v_{\mathrm{s}}\left(\mathrm{CH}_{2}\right)$ mode for dry CER[EOS]. 


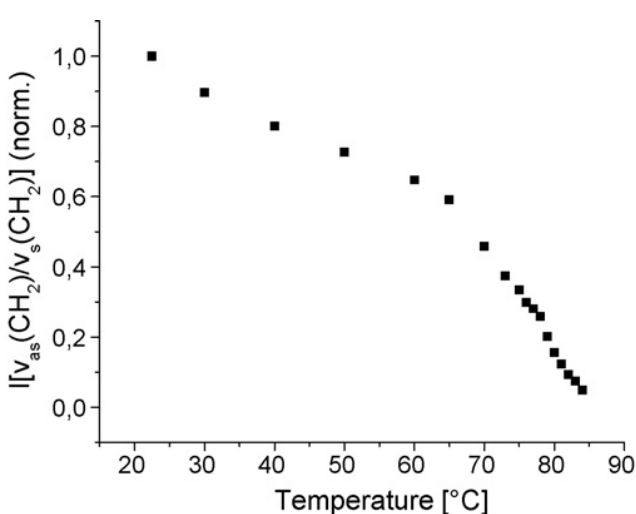

Fig. 9. Temperature dependence of the intensity ratio I $\left[v_{\mathrm{as}}\left(\mathrm{CH}_{2}\right) / v_{\mathrm{s}}\left(\mathrm{CH}_{2}\right)\right]$ for dry CER[EOS].

Raman: $90^{\circ} \mathrm{C}$ ) could be attributed to the different temperature regimes of both methods.

The intensity ratio I $\left(v_{\text {as }}\left(\mathrm{CH}_{2}\right) / \nu_{\mathrm{s}}\left(\mathrm{CH}_{2}\right)\right)$ can be taken as a measure of the relative population of the trans and gauche conformers and represents therefore the degree of the order of the alkyl chain (Wegener et al., 1996; Wang et al., 2000). Fig. 9 shows the temperature dependence of this ratio. It is clearly visible that with increasing temperature the intensity ratio $\mathrm{I}\left(v_{\mathrm{as}}\left(\mathrm{CH}_{2}\right) / v_{\mathrm{s}}\left(\mathrm{CH}_{2}\right)\right)$ continuously decreases and tends to zero at the phase transition temperature.

Summarising, Raman spectroscopy studies on anhydrous CER[EOS] reveal one phase transition from a crystalline to a liquid phase. The alkyl chains are not highly ordered due to the extremely mismatch in the length of the acyl-chain residue. The latter causes a distinct fluidity of the chains preventing the formation of order as being known for many other shorter chain ceramides (Raudenkolb et al., 2003a, 2005b).

Furthermore, anhydrous CER[EOP] was also studied by Raman spectroscopy. In Fig. 10 the Raman spectra at selected temperatures are presented. In accordance with anhydrous CER[EOS], the spectra show neither $\mathrm{LAM}$ nor $\mathrm{CH}_{3}$ rocking mode indicating that the $\mathrm{C}-\mathrm{C}$ bonds at the end of the lipid chains of $\omega$-acylceramide CER[EOP] do not exhibit trans-trans sequence. Furthermore, the splitting of the $\mathrm{CH}_{2}$ scissoring mode into a doublet indicates an orthorhombic chain packing.

From the position of the $\mathrm{CH}_{2}$ symmetric stretching at $2846.9 \mathrm{~cm}^{-1}$ it can be concluded that the hydrocarbon chains are not perfectly ordered. In contrast to CER[EOS], the temperature

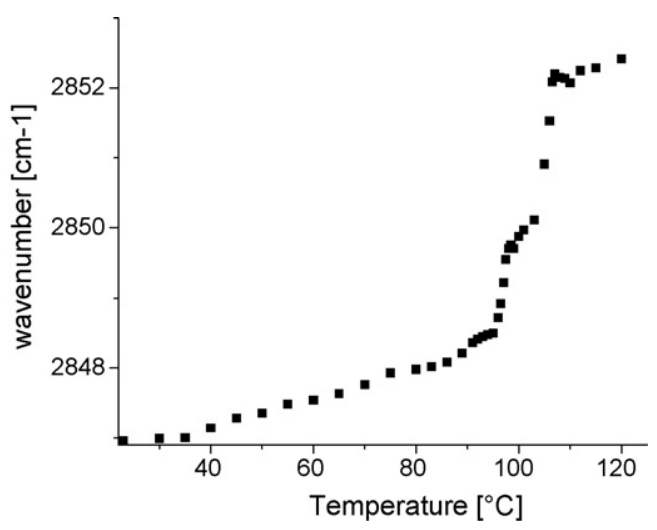

Fig. 11. Temperature dependence of the band position of the $v_{\mathrm{s}}\left(\mathrm{CH}_{2}\right)$ mode for dry CER[EOP].

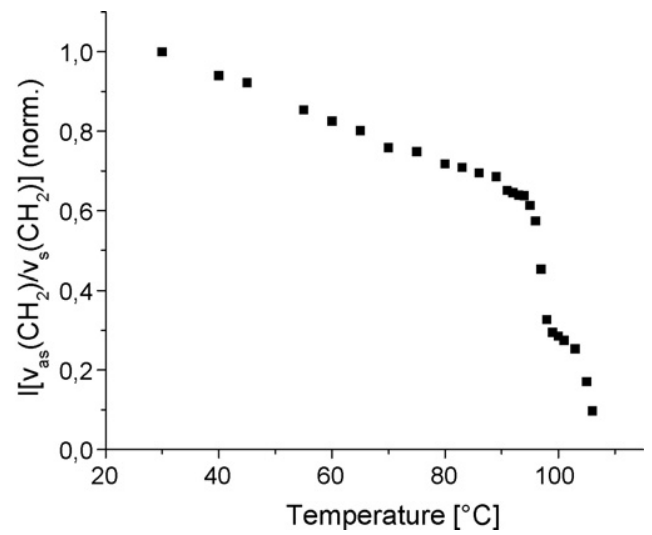

Fig. 12. Temperature dependence of the intensity ratio. $\mathrm{I}\left[\mathrm{vas}_{\mathrm{as}}\left(\mathrm{CH}_{2}\right) / v_{\mathrm{s}}\left(\mathrm{CH}_{2}\right)\right]$ for dry CER[EOP].

dependence of the band position of $v_{\mathrm{s}}\left(\mathrm{CH}_{2}\right)$ reveals two clear jumps at 98 and $105^{\circ} \mathrm{C}$ (Fig. 11).

This partial melting of the chains could be related to a proposed phase transition from orthorhombic to hexagonal chain packing, indicated by the decrease of the lattice distortion observed in X-ray diffraction studies using anhydrous CER[EOP]. The second transition indicates the complete melting because the final position of the $v_{\mathrm{s}}\left(\mathrm{CH}_{2}\right)$ mode at $2853 \mathrm{~cm}^{-1}$ shows the complete melting with loss of order of the chains.
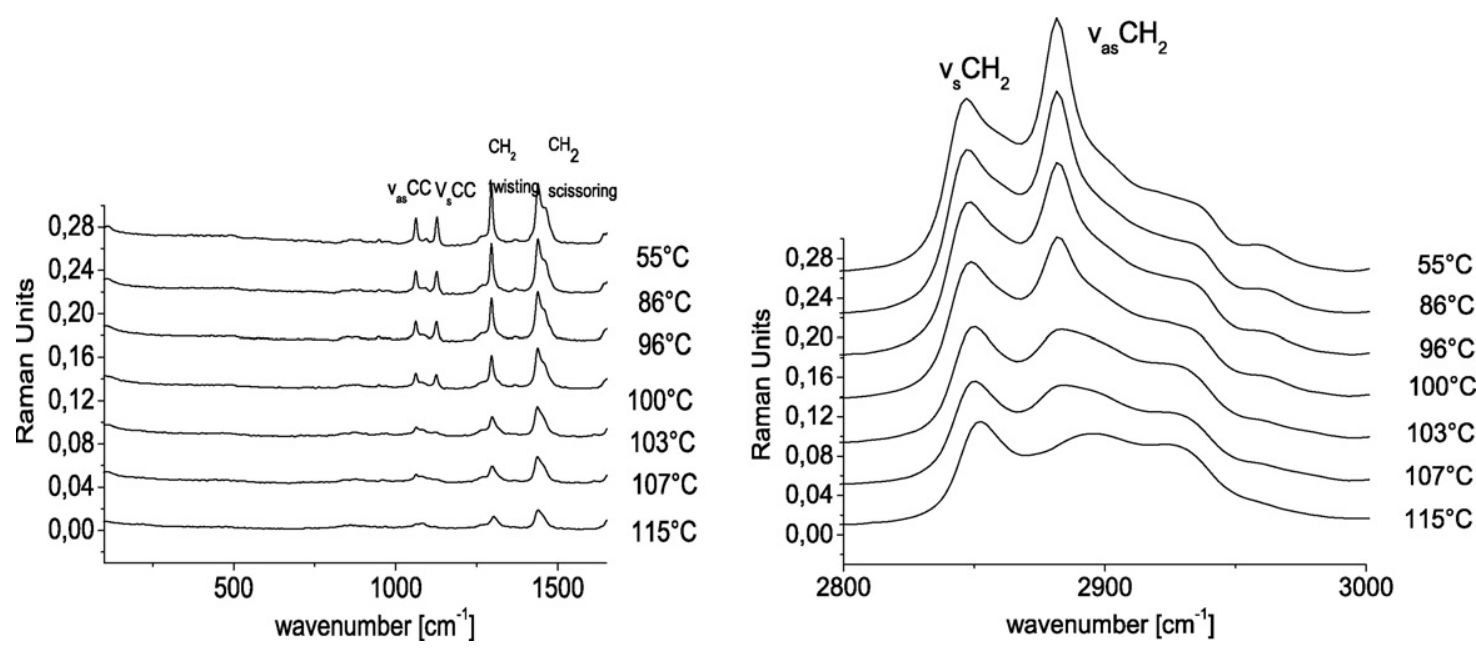

Fig. 10. Raman spectra of dry CER[EOP] in the spectral ranges: (left) $100-1650 \mathrm{~cm}^{-1}$ and (right) $2800-3000 \mathrm{~cm}^{-1}$ taken at different temperatures (indicated). 
To compare the trans-gauche ratio, the quotient of the integrated intensity of the $v_{\mathrm{as}}\left(\mathrm{CH}_{2}\right)$ and the $v_{\mathrm{s}}\left(\mathrm{CH}_{2}\right)$ bands was calculated. Fig. 12 shows a steady rise of the fraction of gauche conformers resulting in a loss of order. In the temperature regions of the phase transitions, two clear jumps occur and in the region of the melting point $\left(T=105^{\circ} \mathrm{C}\right)$, the fraction of the trans conformers tends to zero.

\section{Conclusion}

The present study will contribute to the knowledge about the function of ceramides as main lipid constituents of the stratum corneum. The $\omega$-acylceramides are expected to play a predominant role in the formation of the SC barrier function. But due to the limited availability of these sensitive lipids, their effects are rarely studied until now.

As mentioned above, based on X-ray diffraction studies of SC lipid model systems, CER[EOS] was regarded as a prerequisite for the formation of the LPP with a repeated distance of $13 \mathrm{~nm}$. The substitution of CER[EOS] by the more polar CER[EOP] revealed that the head group structure has a remarkable influence on the behaviour and structure of the SC lipids which results in a reduction of the LPP and in a phase separation (Bouwstra et al., 2000; Jager et al., 2004). According to the present results, the physical-chemical characterization of the $\omega$-acylceramides CER[EOS] and CER[EOP] reveal no pronounced polymorphism, which is observed as a significant feature of shorter chain ceramides (Shah et al., 1995; Raudenkolb et al., 2005a,b). The phase behaviour of both ceramides is strongly influenced by the extreme chain mismatch due to the very long acyl-chain residue. The latter has a much stronger influence compared with the structure of the polar head group, which is discussed as extremely important for the appearance of a rich polymorphism (Wartewig and Neubert, 2007).

However, the additional OH-group of the phyto-sphingosine type CER[EOP] influences the lamellar repeat distance and the chain packing. The less polar, sphingosine type CER[EOS] is more influenced by the long acyl-chain residue. Hydration is necessary for the formation of an extended hydrogen-bonding network between the polar head groups, resulting in the presence of a LPP. In contrast, the more polar CER[EOP] forms the LPP with densely packed alkyl chains already in dry state.

From the results observed with $\omega$-acylceramides used to create a multilamellar structure it cannot be concluded that a skin lipid mixture (a defined crowd of lipids corresponding to the composition of i.e. human skin lipids) induces the formation of a LPP in a similar way (the $\omega$-acylceramide unaffected by other skin lipids). According to literature, the organization of the multilamellar SC lipid (model) membranes is influenced by a lot of factors (Swartzendruber et al., 1989; White et al., 1988; Bouwstra et al., 1991; Garson et al., 1991; Al-Amoudi et al., 2005; Charalambopoulou, 2004; Kessner et al., 2008).

Skin lipid mixtures corresponding to the composition of i.e. human skin are consisting of many species (ceramides, fatty acids and cholesterol). In our studies only single ceramides were used (either in dry state or in aqueous dispersion). This is only a preliminary study and has to be extended to mixed systems. Therefore, no direct conclusion about the phase behaviour of mixed systems can be deduced from the behaviour of a single component.

Further studies using both ceramides are in progress in order to deepen the knowledge about $\omega$-acylceramides as essential skin lipids.

\section{Acknowledgements}

The authors would like to thank Evonik Goldschmidt GmbH (Essen, Germany) for CER[EOS] and CER[EOP]. Financial assistance of the Deutsches Elektronen Synchrotron DESY (Hamburg, Germany) is gratefully acknowledged. D. Kessner would like to thank the Graduiertenförderung des Landes Sachsen-Anhalt for funding. The authors thank A. Wille and B. Arbter for support in chemical purification procedures of CER[EOS] and CER[EOP].

\section{References}

Al-Amoudi, A., Dubochet, J., Norlén, L., 2005. Nanostructure of the epidermal extracellular space as observed by cryo-electron microscopy of vitreous sections of human skin. J. Invest. Dermatol. 124, 764-777.

Bouwstra, J.A., Peschier, L.J.C., Brusse, J., Bodde, H., 1989. Effect of Nalkylazocycloheptan-2-ones including Azone on the thermal behaviour of human stratum corneum. Int. J. Pharm. 52, 47-54.

Bouwstra, J.A., Gooris, G.S., Van der Spek, J.A., Bras, W.W., 1991. Structural investigations of human stratum corneum by small angle X-ray scattering. J. Invest. Dermatol. 97, 1005-1012.

Bouwstra, J.A., Dubbelaar, F.E., Gooris, G.S., Ponec, M., 2000. The lipid organisation in the skin barrier. Acta Derm. Venerol. Suppl. 208, 23-30.

Bouwstra, J.A., Gooris, G.S., Dubbelaar, F.E., Ponec, M., 2002. Phase behavior of stratum corneum lipid mixtures based on human ceramides: the role of natural and synthetic ceramide 1. J. Invest. Dermatol. 118, 606-617.

Bouwstra, J.A., Ponec, M., 2006. The skin barrier in healthy and diseased state. A review. Biochim. Biophys. Acta 1758, 2080-2095.

Bringezu, F., Dobner, B., Brezesinski, G., 2002. Generic phase behavior of branchedchain phospholipid monolayers. Chem. Eur. J. 8, 3203-3210.

Charalambopoulou GC, Steriotis http://www.sciencedirect.com/science?_ob=

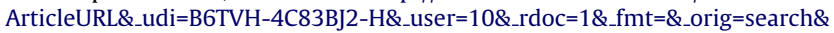
_sort=d\&view=c\&_acct=C000050221\&_version=1\&_urlVersion=0\&_userid=10 \&md5=57e7ca465491375efc715f6311096474 - affa TA, Hauss T, Stubos http://www.sciencedirect.com/science?_ob=ArticleURL\&_udi=B6TVH-4C83BJ2H\&_user=10\&_rdoc=1\&_fmt=\&_orig=search\&_sort=d\&view=c\&_acct=C00005022 \&_version=1\&_urlVersion=0\&_userid=10\&md5=57e7ca465491375efc715f6 311096474 - affa AK, Kanellopoulos http://www.sciencedirect.com/science?_ob $=$ ArticleURL\&_udi=B6TVH-4C83BJ2-H\&_user=10\&_rdoc=1\&_fmt=\&_orig=search \&_sort $=\mathrm{d} \&$ view $=$ c\&_acct $=$ C000050221\&_version=1\&_urlVersion=0\&_userid= $10 \&$ md5=57e7ca465491375efc715f6311096474 - affa NK: Structural alterations of fully hydrated human stratum corneum. Physica B: Condens. Matter, 350, 1-3, Suppl. 1, July 2004, pp. E603-E606.

Chen, H.C., Mendelsohn, R., Rerek, M.E., Moore, D.J., 2000. Fourier transform infrared spectroscopy and differential scanning calorimetry studies of fatty acid homogeneous ceramide 2. Biochim. Biophys. Acta, Biomem. 1468, 293-303.

Coderch, L., Lopez, O., de la Maza, A., Parra, J.L., 2003. Ceramides and skin function. Am. J. Clin. Dermatol. 4, 107-129.

Elias, P.M., 1983. Epidermal lipids, barrier function, and desquamation. J. Invest. Dermatol. 80, 44s-49s.

Friberg, S.F., Osborne, D.W., 1985. Small angle X-ray diffraction patterns of stratum corneum and a model structure for its lipids. J. Dispers. Sci. Technol. 6, 485-495.

Garson, J.C., Doucet, J., Leveque, J.L., Tsoucaris, G., 1991. Oriented structure in human stratum corneum revealed by X-ray diffraction. J. Invest. Dermatol. 96, 43-49.

Gray, G., Yardley, H., 1975. Different populations of pig epidermal cells: isolation and lipid composition. J. Lipid Res. 16, 441-447.

Gray, G.M., White, R.J., 1978. Glycosphingolipids and ceramides in human and pig epidermis. J. Invest. Dermatol. 70, 341.

Hatta, I., Ohta, N., Ban, S., Tanaka, H., Nakata, S., 2001. X-ray diffraction study on ordered, disordered and reconstituted intercellular lipid lamellar structure in stratum corneum. Biophys. Chem. 89, 239-242.

Holleran, W.M., Man, M.Q., Gao, W.N., Menon, G.K., Elias, P.M., Feingold, K.R., 1991. Sphingolipids are required for mammalian epidermal barrier function. Inhibition of sphingolipid synthesis delays barrier recovery after acute perturbation. J. Clin. Invest. 88, 1338-1345.

Jager, M.W., Gooris, G.S., Ponec, M., Bouwstra, J.A., 2005. Lipid mixtures prepared with well-defined synthetic ceramides closely mimic the unique stratum corneum lipid phase behavior. J. Lipid Res. 46, 2649-2656.

Jager, M.W., Gooris, G.S., Ponec, M., Bouwstra, J.A., 2004. Acylceramide head group architecture affects lipid organization in synthetic ceramide mixtures. J. Invest. Dermatol. 123, 911-916.

Kessner, D., Kiselev, M.A., Dante, S., Hauss, T., Lersch, P., Wartewig, S., Neubert, R.H.H., 2008. Arrangement of CER[EOS] in a stratum corneum lipid model matrix-new aspects revealed by neutron diffraction studies. Eur. Biophys. J. Biophys. Lett. 37, 989-999.

Kirchoff, W.H., Levin, I.W., 1987. Description of the thermotropic behaviour of membrane bilayers in terms of Raman spectral parameters: a two state model. J. Res. Natl. Bur. Stand. 92 (2), 113-128.

Lafleur, M., 1998. Phase behaviour of model stratum corneum lipid mixtures: an infrared spectroscopy investigation. Can. J. Chem. 76, 1501-1511.

Mizutani, Y., Mitsutake, S., Tsuji, K., kihara, A., Igarashi, Y., 2009. Ceramide biosynthesis in keratinocyte and its role in skin function. Biochimie 91, 784-790.

McIntosh, T.J., Stewart, M.E., Downing, D.T., 1996. X-ray diffraction analysis of isolated skin lipids: reconstruction of intercellular lipid domains. Biochemistry 35 , 3649-3653.

McIntosh, T.J., 2003. Organization of skin stratum corneum extracellular lamellae: diffraction evidence for asymmetric distribution of cholesterol. Biophys. J. 85, 1675-1681. 
McPhail, R.A., Strauss, H.L., Snyder, R.G., Elliger, C.A., 1984. C-H-stretching modes and the structure of n-alkyl-chains. 2. Long, all-trans chains. J. Chem. Phys. 84, 334-341.

Mendelsohn, R., Moore, D.J., 1998. Vibrational spectroscopic studies of lipid domains in biomembranes and model systems. Chem. Phys. Lipids 96, 141-157.

Moore, D.J., Rerek, M.E., Mendelsohn, R., 1997. FTIR spectroscopy studies of the conformational order and phase behavior of ceramides. J. Phys. Chem. B101, 8933-8940.

Neubert, R., Rettig, W., Wartewig, S., Wegener, M., Wienhold, A., 1997. Structure of stratum corneum lipids as characterized by Fourier transform Raman spectroscopy and DSC. II. Mixtures of ceramides and saturated fatty acids. Chem. Phys. Lipids 89, 3-14.

Raudenkolb, S., Hübner, W., Rettig, W., Wartewig, S., Neubert, R., 2003a. Polymorphism of ceramide 3. Part 1: an investigation focused on the head group of N-octadecanoylphytoshingosine. Chem. Phys. Lipids 123, 9-17.

Raudenkolb, S., Wartewig, S., Brezesinski, G., Funari, S.S., Neubert, R.H.H., 2005b. Hydration properties of $\mathrm{N}$ - $(\alpha$-hydroxyacyl)-spingosine: X-ray powder diffraction and FT-Raman spectroscopic studies. Chem. Phys. Lipids 136, 13-22.

Raudenkolb, S., Wartewig, S., Neubert, R., 2003b. Polymorphism of ceramide 3. Part 2: a vibrational spectroscopic and X-ray powder diffraction investigation of $\mathrm{N}$ octadecanoyl phytosphingosine and the analogous specifically deuterated d35 derivative. Chem. Phys. Lipids 124, 89-101.

Raudenkolb, S., Wartewig, S., Neubert, R., 2005a. Polymorphism of ceramide 6. A vibrational spectroscopic and X-ray powder diffraction investigation of the diastereomers of $\mathrm{N}$-( $\alpha$-hydroxyoctadecanoyl)-phytosphingosine. Chem. Phys. Lipids 133, 89-102.

Shah, J., Atienza, J.M., Duclos, R.I., Rawlings, A.V., Dong, Z., Shipley, G., 1995. Structural and thermotropic properties of synthetic C16:0 (palmitoyl) ceramide: effect of hydration. J. Lipid Res. 36, 1936-1944.
Snyder, R.G., Scherer, J.R., 1979. Band structure in the $\mathrm{CH}$ stretching region of the Raman spectrum of extended polymethylene chain: Influence of Fermi resonance. J. Chem. Phys. 71, 3221-3228.

Snyder, R.G., Hsu, S.L., Krimm, S., 1982. Vibrational spectra in the C-H stretching region and the structure of the polymethylene chain. Spectrochim. Acta 34A, 395-406.

Swartzendruber, D.C., Wertz, P.W., Kitko, D.J., Madison, K.C., Downing, D.T., 1989. Molecular models of the intercellular lamellae in mammalian stratum corneum. J. Invest. Dermatol. 92, 251-257.

Wang, R., Guo, J., Baran, G., Wunder, S.L., 2000. Characterization of the state of order of octadecysilane chains on fumed silica. Langmuir 16, 568-576.

Wartewig, S., Neubert, R., Rettig, W., Hesse, K., 1998. Structure of stratum corneum lipids characterized by FT-Raman spectroscopy and DSC. IV. Mixtures of ceramides and oleic acid. Chem. Phys. Lipids 91, 145-152.

Wartewig, S., Neubert, R.H.H., 2007. Properties of Ceramides and their impact on the stratum corneum structure - A review - Part 1: Ceramides. Skin Pharmacol. Physiol. 20, 220-229.

Wegener, M., Neubert, R., Rettig, W., Wartewig, S., 1996. Structure of stratum corneum lipids characterized by FT-Raman spectroscopy and DSC. I. ceramides. Int. J. Pharm. 128, 203-213.

Wegener, M., Neubert, R., Rettig, W. Wartewig, S., 1997. Structure of stratum corneum lipids characterized by FT-Raman spectroscopy and DSC. III. Mixtures of ceramides and cholesterol. Chem. Phys. Lipids 88, 73-82.

Wertz, P.W., van den Bergh, B., 1998. The physical, chemical and functional properties of lipids in the skin and other biological barriers. Chem. Phys. Lipids 91 85-96.

White, S., Mirejosray, D., King, G., 1988. Structure of lamellar domains and corneocytes envelopes in murine stratum corneum. Biochemistry 27, 37253732. 